\title{
SÉPTIMO ENCUENTRO DE PROFESORES DE CIENCIAS DE LA SALUD
}

\author{
Universitat de Barcelona. Facultad de Farmacia \\ Campus Pedralbes
}

30 de enero - 1 de febrero de 2013

\begin{tabular}{|c|c|}
\hline \multicolumn{2}{|c|}{ COMITÉ ORGANIZADOR } \\
\hline $\begin{array}{l}\text { Presidente: } \\
\text { Presidenta honorífica: } \\
\text { Vicepresidenta: } \\
\text { Secretaria: } \\
\text { Vocales: }\end{array}$ & $\begin{array}{l}\text { Esteva de Sagrera, Juan } \\
\text { Barbé Rocabertt, Coloma } \\
\text { Pallàs Lliberia, Mercè } \\
\text { Halbaut Bellowa, Lyda } \\
\text { Amat Miralles, Concepción } \\
\text { Aróztegui Trenchs, Montserrat } \\
\text { Arqué Blanco, Mercè } \\
\text { Manzanares Céspedes, Ma Cristina } \\
\text { Palma Linares, Imma } \\
\text { Rius Bofill, Núria } \\
\text { Turbany Oset, Jaume } \\
\text { Vallès Segalés, Antoni } \\
\text { Vidal Carou, Ma Carmen } \\
\text { Zalacaín Vicuña, Antonio J. }\end{array}$ \\
\hline
\end{tabular}

\section{COMITÉ CIENTíFICO}

Presidente:

Vocales:
Prats Climent, Baldiri

Buxaderas Sánchez, Susana

Götzens García, Victor

Gracenea Zugarramurdi, Mercedes

Honrubia Pérez, María

Miñarro Carmona, Montserrat

Sánchez González, Silvia

\section{SECRETARÍA TÉCNICA}

M. ${ }^{a}$ Pilar Vaquero Serraima, Anna Brillas Sancliments y Paola Bustos Salgado

\section{Organizan:}

Facultad de Farmacia (Farmacia, Ciencia y Tecnología de los Alimentos, Nutrición Humana y Dietética), Facultad de Psicología, Facultad de Medicina (Medicina, Ciencias Biomédicas e Ingeniería), Facultad de Odontología, Escuela Universitaria de Enfermería (Enfermería y Podología).

\section{Colaboran:}

ADMES-Barcelona, SEDEM, SCB, SAE, AEDN 


\title{
Presentación
}

\author{
Baldiri Prats Climent
}

Ya estamos en el Séptimo Encuentro de Profesorado de Ciencias de la Salud de la Universidad de Barcelona. Las ediciones anteriores han permitido la consolidación de varios aspectos relacionados con la gran transformación que ha tenido lugar en los últimos años en los estudios universitarios. En su inicio, estos encuentros sirvieron para dar a conocer las nuevas tecnologías y para ver cómo los proyectos de innovación docente iban ofreciendo la posibilidad de incorporar nuevas estrategias a nuestra actividad educativa.

Estos encuentros han servido también para ayudarnos a entender el famoso 'Plan Bolonia' y todo lo que ha comportado la incorporación al Espacio Europeo de Educación Superior; las distintas aportaciones, mesas redondas y conferencias han permitido entender mejor la evaluación continuada, las competencias, el trabajo presencial, el trabajo dirigido..., digerir este cambio tan importante que nos ha acompañado en los últimos años. Seguro que en este encuentro o en las próximas ediciones nos cuestionaremos si hemos hecho lo correcto, si ha servido realmente para mejorar nuestra docencia, al fin y al cabo, si hemos conseguido formar mejores profesionales.

Sin duda, esta séptima edición debe tratar también la situación general de la sociedad y su repercusión en el ámbito universitario. La calidad en tiempo de crisis será el tema que abrirá el programa y seguro que su contenido seguirá planeando sobre el resto de los temas de actualidad que serán tratados. Estamos viviendo momentos difíciles, el futuro es incierto y, además, Coloma Barbé, impulsora de estos encuentros, nos ha dejado. Será un encuentro extraño sin ella. Echaremos de menos no sólo su sonrisa, sino también su vitalidad, su dedicación y sobre todo su amistad, pero precisamente por ella tenemos que conseguir que este séptimo encuentro sea todo un éxito.

Se ha intentado conseguir un programa equilibrado en el que, a pesar de que el tema principal sigue siendo el intercambio de experiencias docentes y las iniciativas de innovación, se actualizarán otros contenidos como la relación entre ciclos formativos y universidad, el trabajo de fin de grado o la integración de la investigación con la docencia. Las otras vertientes de la actividad del profesorado, la investigación y la gestión, también se van a abordar en mesas específicas para incluirlas en el contexto docente.

No quiero acabar sin mostrar agradecimiento a todos los compañeros que han colaborado con la presentación de sus trabajos, por el esfuerzo realizado y por su generosidad al compartir sus experiencias esperando que, como siempre, nos ayuden a mejorar nuestra estimada actividad docente.
Presidente del Comité Científico. 